\title{
Effects of flow parameters on the performance of vertical axis swirling type Savonius wind turbine
}

\author{
A. Al-Faruk* and A. S. Sharifian \\ Computational Engineering and Science Research Centre \\ University of Southern Queensland, Toowoomba 4350 Australia \\ *Email: U1031944@umail.usq.edu.au, alfaruk.bd@gmail.com
}

Phone: +61 74631 2633; Fax: +61 746312110

\begin{abstract}
Wind tunnel experiments were carried out on a geometrically optimal swirling Savonius turbine by varying flow parameters to determine their effects on power and torque coefficients. The optimum geometrical configuration used in this experiment was adopted from an earlier study that features 0.20 blade overlap ratio, $195^{\circ}$ blade arc angle, and 1.06 rotor aspect ratio of a $320 \mathrm{~mm}$ diameter rotor with closed top end plate. The parameters considered in this experiment are the hot air temperature, hot air mass flow rate, hot air inlet diameter of swirl chamber, and the free-stream wind velocity. The results indicate that higher hot air temperature and hot air mass flow rate enhance the performance of the turbine while power coefficient reached maximum at $16 \mathrm{~mm}$ diameter of hot air inlet. Tests on the optimum geometry at five wind velocities revealed that power coefficients are higher in higher wind velocities, which suggests that swirling flow becomes stronger with the increase of wind velocity.
\end{abstract}

Keywords: Savonius wind turbine; wind tunnel; split channel; swirling flow; power coefficient.

\section{INTRODUCTION}

Many countries are seeking to mitigate global warming issues by reducing our reliance on fossil fuels for energy generation [1], which is the main source of greenhouse gas emissions. The recent increase in fossil fuel cost is paving the way for new technology to harness energy from renewable energy sources. Among the renewable sources, abundant and freely available solar and wind can be used as an alternative source to provide clean and environmentally friendly power [2]. Being an energy source of low impact on environment, with low effects on health and negligible safety issues, demand for wind-power has been increasing exponentially in recent years $[3,4]$. However, the main challenge of using wind energy for stand-alone power system applications is the availability of power meeting the daily and seasonal patterns of load demand. Until now, the propeller type horizontal axis wind turbines (HAWTs) have played a primary role in response to this demand. The HAWTs can provide large power outputs, but need greater wind velocities and often generate low-frequency noise that can be harmful [5]. In contrast, vertical axis wind turbines (VAWT) are isolated from these environmental problems, resulting in the recent expansion of their use in urban environments [6]. Among a number of VAWTs, the Savonius turbine is known as the most quiet wind power source as it runs at lowest tipspeed ratio [7]. Therefore, it can be employed to generate on-site electricity in city environments. The turbine also easily starts to rotate at low wind speeds because the 
differential drag on the two blades produces the torque. This allows the turbine to rotate not only at the top of high buildings but also at street level in a city. As there are no severe restrictions on blade material, the manufacturing process is easy to realize on-site using onsite materials [8]. The downside is that Savonius turbines are widely considered as dragdriven devices [9]. The power extraction efficiency (power coefficient, $C_{p}$ ) from the wind is typically less than half of the efficiency of HAWTs. Experiments on conventional Savonius rotors at an overlap ratio of 0.15 and an aspect ratio of 1.0 have been reported to have a maximum coefficient of power of $17.3 \%$ as reported by Fujisawa and Gotoh [10] and $17.0 \%$ by Kamoji, Prabhu [11] when tested in an open jet wind tunnel. This value corresponds to only one third of the Betz's limit of 59.3\%. However, it has been observed that at low angles of attack, the lift force also contributes to the overall torque generation $[12,13]$. Thus, it can be concluded that the Savonius rotor is a combination of a drag-driven and lift-driven device. Therefore, it can go beyond the limit of power coefficient $\left(C_{p}\right)$ of purely drag-driven machines, which is $8 \%$ [14].

Continual efforts are being made to improve the coefficient of power either by examining the effects of various design parameters or by incorporating additional features, or by modifying the shape of the conventional Savonius rotor. Investigations in open jet wind tunnel showed that the three-bladed rotor is inferior to the two-bladed rotor while the performance of two-stage rotor is superior to the single-stage rotor [15]. Akwa, Júnior [12] numerically investigated the blade overlap ratio to find out an optimum rotor and obtained best performance at 0.15 . An investigation on a modified Savonius rotor with central shaft between the end plates was reported by Modi and Fernando [13] to have a maximum power coefficient of around 32\% based on closed jet wind tunnel testing. Kamoji, Kedare [16] examined the influence of the end plates and the central shaft on a single stage modified Savonius rotor and found that the power coefficient rose up to $21 \%$ with the best combination. Saha and Rajkumar [17] confirmed that the twisted blades in the vertical direction provide a better performance than conventional cylindrical blades.

Another area of improvement is the introduction of wind collection equipment around the turbine presented by Ushiyama, Nagai [18]. Surrounding a turbine with a guide box succeeded in raising the power coefficient by up to $123 \%$ for two-blade and $150 \%$ for threeblade models relative to the original models reported by Irabu and Roy [19]. Ogawa, Yoshida [20] confirmed that the use of a deflecting plate increased the rotor power by approximately $30 \%$ than that of a rotor without a deflecting plate. In a similar manner, further significant success was reported using mobile types of solid curtains that recorded about $38 \%$ increase in power coefficient with optimum curtain arrangement [21]. These large improvements are considered to be due to the drag reduction on the returning blade that migrates upstream against the main flow. Al-Faruk, Sharifian [22] proposed an innovative low cost technique of performance improvement by inducing hot air swirling flows inside the turbine enclosure. They numerically investigated the possibility of combining the fire-whirl mechanism with the primary Savonius wind turbine mechanism by modifying the blade geometry in a new designed configuration (naming the new design as swirling Savonius turbine, SST). They reported that swirling flows were formed in the vortex chamber of the turbine when hot air was used instead of fire and the comparison of power coefficient between the SST and the conventional turbine showed improvement in the performance. Later on, Al-Faruk and Sharifian [23] carried out experimental investigations on the swirling Savonius turbines by considering several geometrical design parameters to find out their effects on the aerodynamic coefficients as well as to optimize the geometry for which the power coefficient reaches the maximum. However, the effect 
of incoming wind velocity, hot air temperature and mass flow rate at the swirling chamber inlet of the new design was not covered in their experimental research.

In the present study, experimental investigations were carried out on the optimum configuration of SST rotor to determine the effects of flow parameters on the aerodynamic coefficients. Several flow parameters such as hot air temperature, hot air mass flow rate, free-stream wind velocity, and the hot air inlet diameter of the swirling chamber are considered in this experiment. The existing arrangement of experimental set-up allowed the performance assessments of the swirling Savonius rotor as well as conventional Savonius rotor in different wind velocities. Performance comparison between the swirling Savonius and the optimum conventional Savonius turbines were made in the same experimental conditions.

\section{SWIRLING SAVONIUS TURBINE}

The swirling Savonius turbine is similar to the classic Savonius turbine, which consists of two identical blades of semi-cylinder like surfaces moving sideways and making an overlap between them, as shown in Figure 1.

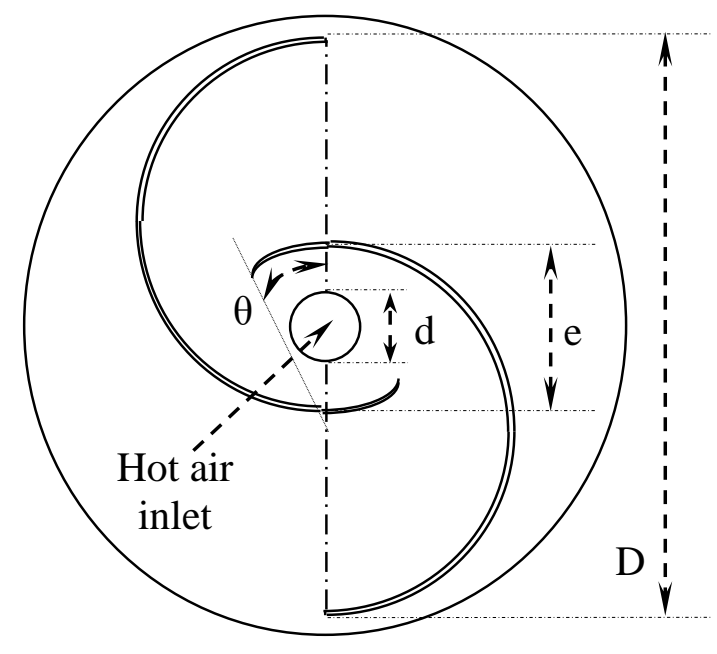

(a)

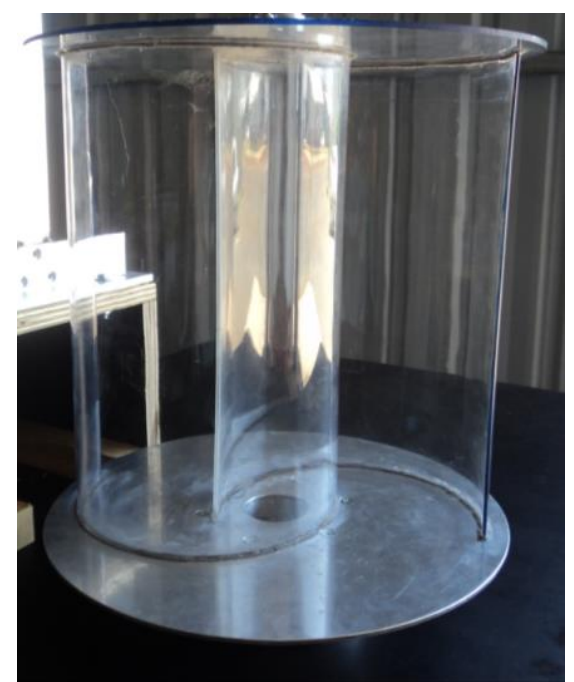

(b)

Figure 1. Swirling Savonius rotor (a) schematic of blade parameters at bottom end plate; (b) photographic view.

The modification made in the SST is that the inner tips of the half cylindrical blades extended further to construct a swirling chamber [22, 23]. This results in larger blade arc angle of the swirling Savonius rotor than the conventional Savonius rotor. The extended angle $(\theta)$ of the blades, as shown in Figure 1, lowers the air entrainment gap of the vortex chamber. A circular hole of diameter $d$ made in the bottom end plate that acts as hot air inlet of the swirling chamber. The blade overlap ratio $(\beta)$ of the SST defined as the ratio of blade overlap distance $(e)$ and the inside diameter $(I D)$ of the blade. The diameter of the rotor can be calculated using the inside and outside diameters of the blade pipe and the blade overlap ratio from $D=O D+I D(1-\beta)$ formula. As the end plate diameter is usually made $10 \%$ larger than the rotor diameter for the conventional Savonius turbine with optimum performance [24], the same ratio was applied to the SST rotors. 


\section{METHODS AND MATERIALS}

Light weight and transparent Acrylic tube of $180 \mathrm{~mm}$ outside diameter and $3 \mathrm{~mm}$ wall thickness was chosen as the blade material. The bottom end plate was made from $3 \mathrm{~mm}$ thick Aluminium sheet and $3 \mathrm{~mm}$ Acrylic sheet was used as the top end plate to provide a clear view of the rotor. Circular holes of $44 \mathrm{~mm}$ and $100 \mathrm{~mm}$ diameters were cut at the centre of the bottom and top end plates, respectively, as shown in Figure 1(b). The bottom hole acted as the hot air inlet of the chamber and the top hole provided an opening to the vortex chamber. Blade shape groves were etched on the end plates to ensure a robust and accurate assembly. After inserting the ends of the blades into the groves of the end plates, strong adhesive was used to complete the fabrication of the rotor. Very thin Aluminium sheets were placed on top of the hot air inlet of swirl chamber to change the diameter during the experiments.

\section{Experimental Set-Up}

The experimental set-up consists of a structural test bench housing the rotor and hot air generation chamber, wind tunnel, and measurement devices. The wind tunnel used in the experiments was an open-jet type driven by a two contra rotating fans. Air exited from a circular outlet of $50 \mathrm{~cm}$ diameter as shown in Figure 2 . The wind velocity could be changed by blocking the passage in the suction side, or with the use of an adjustable damper in the wind tunnel. The rotor and several measuring devices were placed on top of the bench and the heating chamber was placed underneath the test bench.

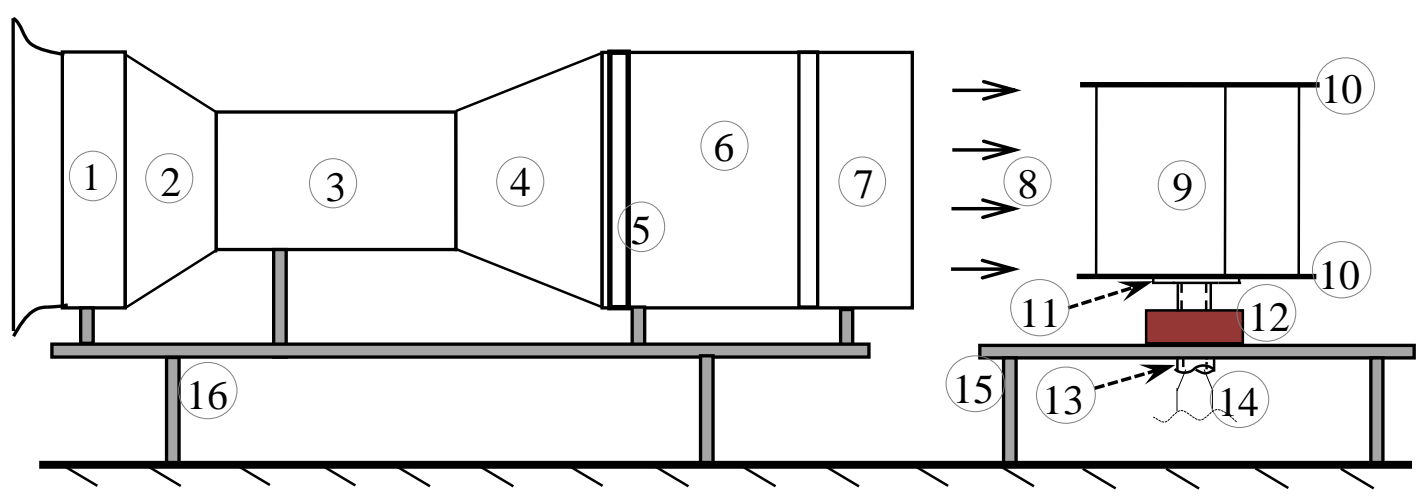

Figure 2. Schematic diagram of the experimental set-up (not to scale): 1-bell mount inlet, 2-contraction cone, 3-closed test section, 4-divergent cone, 5-damper, 6-contra rotating axial fan, 7-outlet, 8-open jet, 9-rotor, 10-end plate, 11-flange, 12-bearing housing, 13-hollow shaft, 14-hot air pipe, 15-test bench, and 16-structure.

The bottom end plate of the turbine was coupled with the end flange of an Aluminium hollow shaft and placed into a ball bearing $(50 \mathrm{~mm}$ bore diameter) which was fixed to the test bench as shown in Figure 2. To reduce bearing friction, the seals were removed and the bearing was washed in petrol to remove the grease before mounting. The bearing was sprayed with a commercially available spray lubricant before each experiment, as suggested by [25] to maintain congruity among the readings. A $50 \mathrm{~mm}$ diameter Stainless Steel pipe was used as a chimney that carried the hot air from the hot air enclosure and into the swirling chamber. The heating zone at the bottom of the test table was fabricated using $0.9 \mathrm{~mm}$ Stainless Steel sheet. Two cooking hot plates were used to heat the air. Only hot surfaces of the heating plates sat inside the enclosure due to safety concerns as shown in 
Figure 3. To minimize heat loss to the environment from the hot air enclosure and the chimney, room insulation batts were used to cover outer surfaces. A $250 \mathrm{~mm} \times 200 \mathrm{~mm}$ opening with sliding cover was made in the side wall to allow and control the entry of fresh air into the chamber.

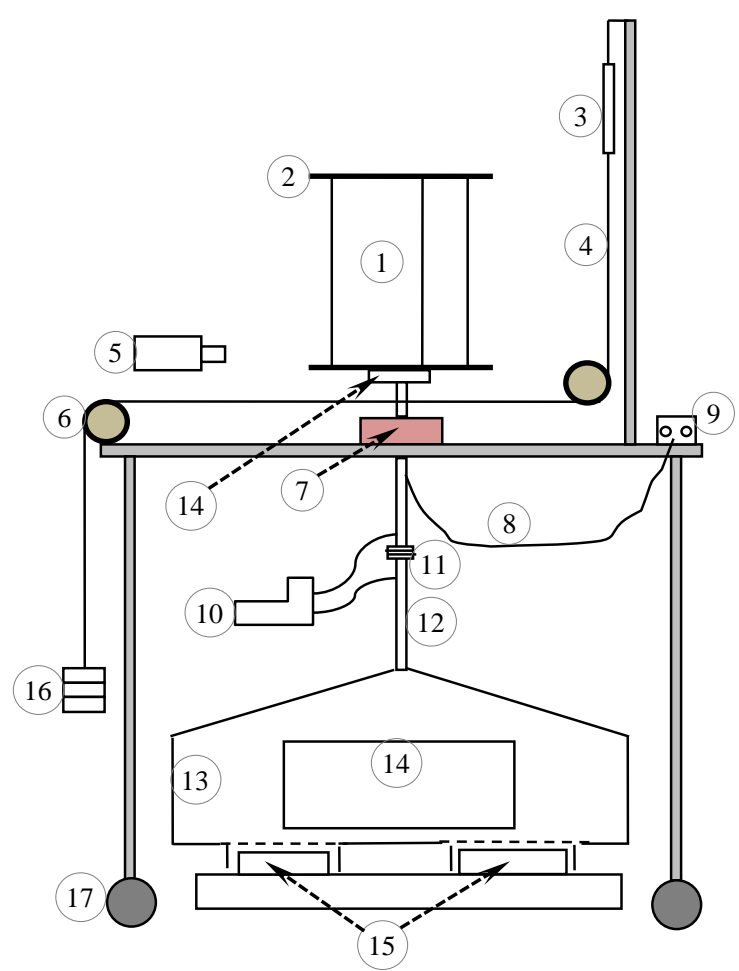

(a)

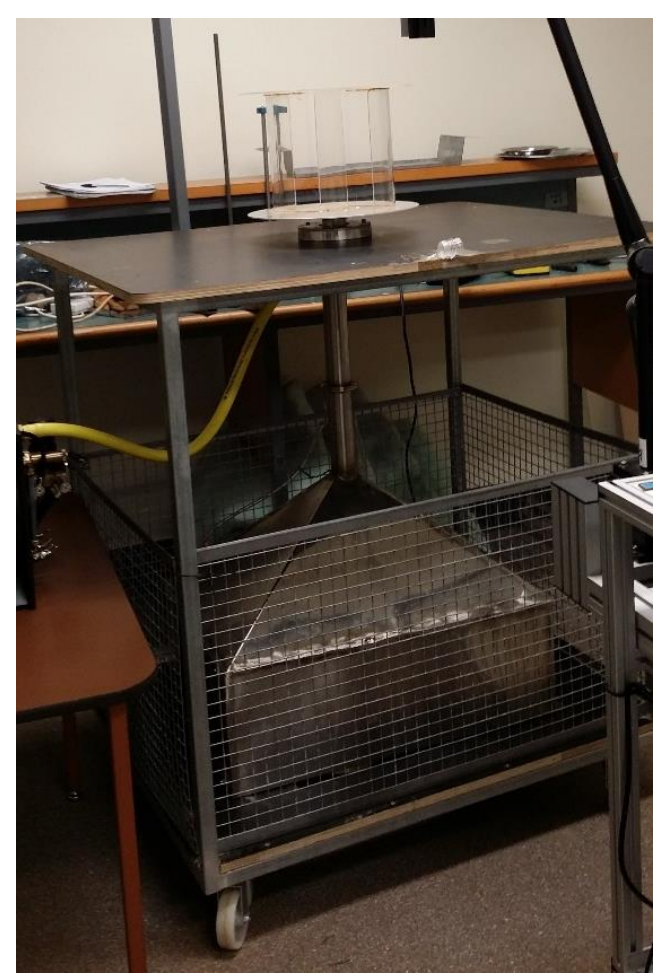

(b)

Figure 3. Experimental test bench (a) schematic diagram: 1-rotor, 2-end plate, 3spring balance, 4-string, 5-photo tachometer, 6-pulley, 7-bearing housing, 8thermocouple, 9-thermocouple reader, 10-monometer, 11-orifice plate, 12-hot air pipe (chimney), 13-hot air enclosure, 14-enclosure inlet, 15-hot plates, 16-dead mass, and 17-castor wheel; (b) photographic view.

A K-type thermocouple was inserted through a hole at the top end of the chimney to measure temperature of the warm air entering the swirling chamber of the rotor. A hot wire anemometer was used to measure wind velocity and a brake rope dynamometer was used to apply brake load on the Savonius rotor and to measure the rotor torque. The brake rope dynamometer consisted of a weighing pan, two pulleys and a spring balance. The weighing pan and the spring balance were connected by a fishing nylon string of $0.4 \mathrm{~mm}$ diameter running over the pulleys. The string was wounded one turn $\left(360^{\circ}\right)$ over the hollow shaft of the rotor on the top of the bearing housing.

\section{Experimental Procedure}

The test bench was placed at $3.06 \mathrm{~m}$ downstream from the wind tunnel exit such that the centre of the rotor was in line with the centre of the tunnel exit to allow sufficient distance for the wind to become uniform at the rotor plane and to minimize experimental error. Steady free stream flow is also necessary for the stability of the swirling flows inside the vortex chamber as well as to reduce the fluctuations of rotor angular velocity. To minimize 
error during the wind velocity measurements, the hot wire anemometer was fixed to the test bench using a stand and G-clamp, as shown in Figure 4(a). Wind velocities at $50 \mathrm{~cm}$ upstream and $50 \mathrm{~cm}$ downstream from the central axis of rotor were measured at nine points located systematically in the projected area of the rotor. Then, the average value of each projected area was averaged to obtain the wind velocity, which was used to calculate the performance parameters. The measured velocity distribution at $50 \mathrm{~cm}$ upstream of the rotor along the central axis was uniform within $\pm 5 \%$, as presented in Figure 4(b).

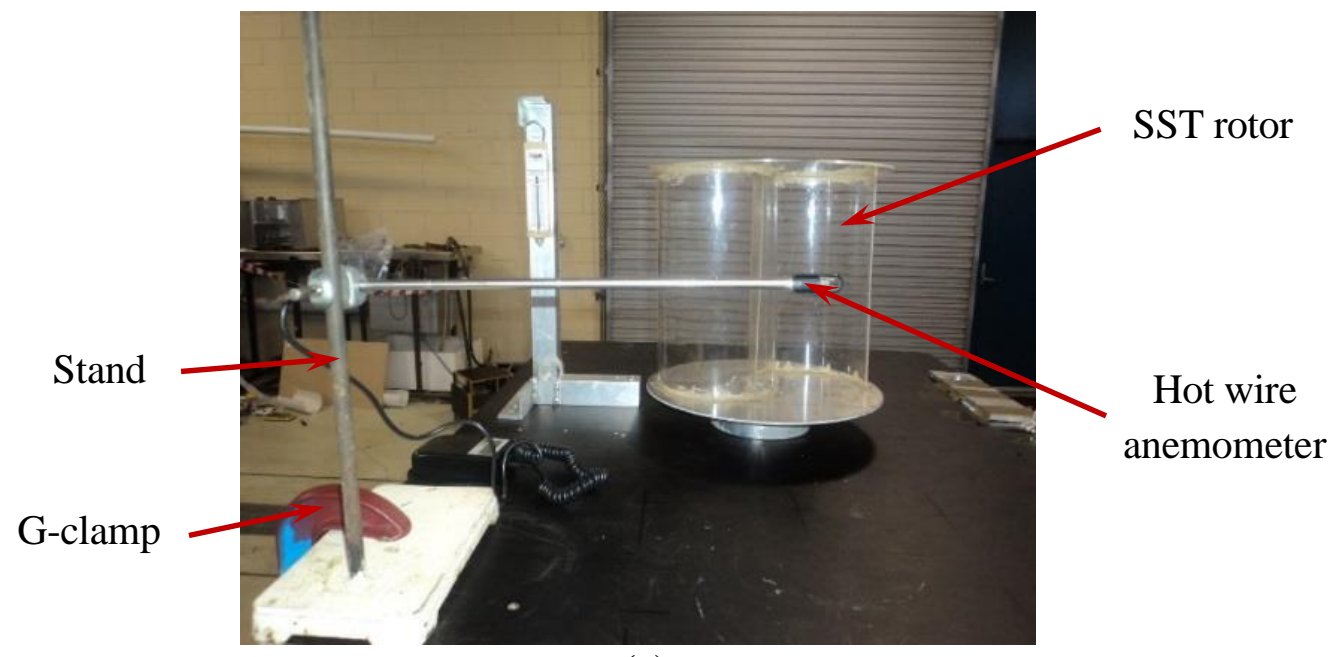

(a)

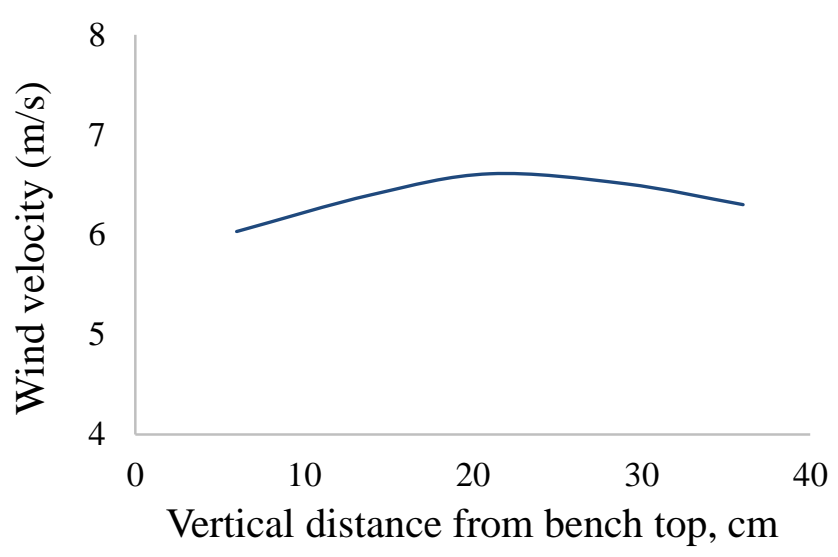

(b)

Figure 4. (a) Wind velocity measurement set-up by hot wire anemometer; (b) velocity distribution at $50 \mathrm{~cm}$ upstream along rotor axis.

The rotors were allowed to rotate from no load condition, and the rotational speed $(\omega)$ is recorded by a digital photo tachometer when the rotor reached a steady state condition. The rotors were loaded gradually (adding 50 gram of mass each time) by the rope brake dynamometer from a no load condition to the highest load that stopped the rotor. The spring balance readings $(S)$ and the dead weights $(W)$ of the dynamometer are recorded and then used to calculate the brake torque of the rotor using the formula

$$
T=\left(\frac{D_{s h}+d_{w}}{2}\right)(W-S)
$$


where $D_{s h}$ is the diameter of the rotor shaft and $d_{w}$ is the string diameter.

The brake power of the rotor can be calculated from the brake torque and the angular velocity $(\omega)$ of the rotor as

$$
P=T \omega
$$

The dimensionless performance parameters commonly used in the aerodynamics of wind turbines are coefficient of power and coefficient of torque. For an incoming wind velocity $U$, the coefficient of power is the ratio of mechanical brake power to wind power

$$
C_{p}=\frac{P}{\frac{1}{2} \rho D H U}
$$

The coefficient of torque is the ratio of applied torque to the wind torque can be presented as

$$
C_{t}=\frac{T}{\frac{1}{4} \rho D^{2} H U^{2}}
$$

The corresponding values of $C_{p}$ and $C_{t}$ are usually presented with or tip speed ratio, $\gamma$ defined as

$$
\gamma=\frac{\omega D}{2 U}
$$

where $\rho$ is the air density, $D$ rotor diameter, and $H$ rotor height.

\section{Uncertainties in Measurements and Experiments}

The relative uncertainties of the independent or measurable parameters in the experimental study were determined first and it was found that the uncertainties were very low except for rotor angular speed $(2.7 \%)$ and wind velocity $(7.1 \%)$. Then, the relative uncertainties of the dependent parameters were estimated based on the functional form of the independent parameters from the general uncertainty formula [26]. The relative uncertainty of the torque and power coefficients were found to be $21.5 \%$ and $21.7 \%$, respectively.

\section{RESULTS AND DISCUSSION}

A series of wind tunnel experiments were carried out on geometrically optimum swirling Savonius turbines by changing the flow parameters. The optimum configuration was adopted from an earlier study by Al-Faruk and Sharifian [23], which featured 0.20 blade overlap ratio, $195^{\circ}$ blade arc angle, and 1.06 rotor aspect ratio of a $320 \mathrm{~mm}$ diameter rotor with closed outlet of swirling chamber. These parameter values of optimum SST rotor give the highest power coefficient in their study. The flow parameters studied in this study were the hot air temperature, hot air mass flow rate, hot air inlet diameter and the free-stream 
wind velocity to determine their effects on power coefficient. The results were analyzed based on the influence of the parameters on the power and torque coefficients.

\section{Effect of Inlet Hot Air Temperature}

The effect of hot air temperature at swirling chamber inlet was investigated on the swirling Savonius turbine at $4.90 \mathrm{~m} / \mathrm{s}$ wind velocity, $16 \mathrm{~mm}$ diameter of hot air inlet, and $66 \%$ opening of hot air enclosure inlet. The hot air temperature was changed in the experiment by controlling the heat settings of the hot plate, which had two cook tops of 900 Watts and 1500 Watts power respectively, with a total of eight heat settings. Experiments were conducted using hot air of different temperatures ranging from $70^{\circ} \mathrm{C}$ to $107^{\circ} \mathrm{C}$ by running the hot plates individually or simultaneously and keeping all the other variables the same.

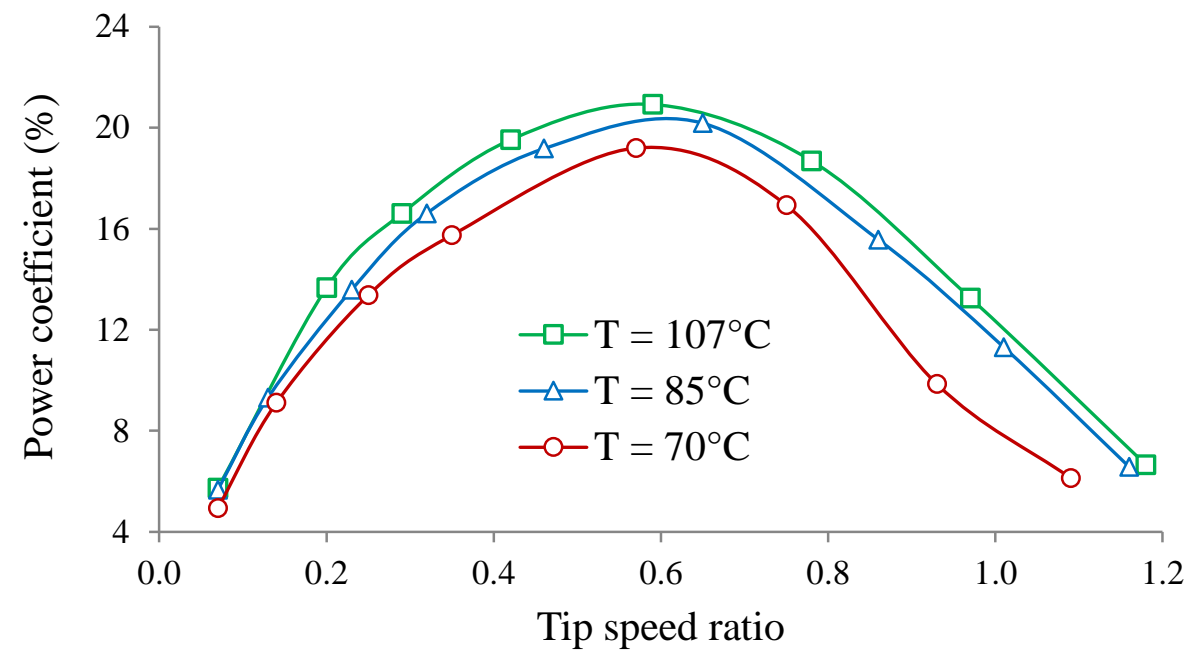

Figure 5. Change of power coefficient with blade tip speed ratio for three hot air temperatures using $16 \mathrm{~mm}$ hot air inlet diameter, $66 \%$ enclosure opening, and $4.9 \mathrm{~m} / \mathrm{s}$ wind velocity.

The dimensionless power coefficient versus tip speed ratio plots are presented in Figure 5 for three different hot air temperatures. The power coefficient increases with the increase of tip speed ratio; reaches maximum at around 0.60 tip speed ratio for all three cases, and then decreases with tip speed ratio. This behaviour is congruent with the power coefficient curves of other wind turbines [27] because at low tip speed ratios, torque is high but angular speed is low and similarly torque is low at high tip speed ratios. As mechanical power is the product of angular speed and torque, the power coefficients-tip speed ratio plots form a symmetric bell-shape curve and reach the maximum at around half of no load tip speed ratio. It is clear from Figure 5 that the power coefficients at $70^{\circ} \mathrm{C}$ hot air temperature are lower than that of the $85^{\circ} \mathrm{C}$ and $107^{\circ} \mathrm{C}$ temperatures, whose values are almost identical. The maximum power coefficient obtained at different hot air temperatures is plotted with respect to their temperatures in Figure 6. The maximum power coefficient increases with the increase of hot air temperature and the maximum power coefficient is $20.92 \%$ found at $107^{\circ} \mathrm{C}$ hot air temperature corresponding to the maximum heater power of 2400 Watts. The strength of the swirling flow induced by split channels depends on the temperature of hot air inlet and the strength could be increased by increasing the inlet temperature [28]. The performance of SST rotor improves with higher inlet temperature because of the strong swirl flow generated at the swirling chamber. 


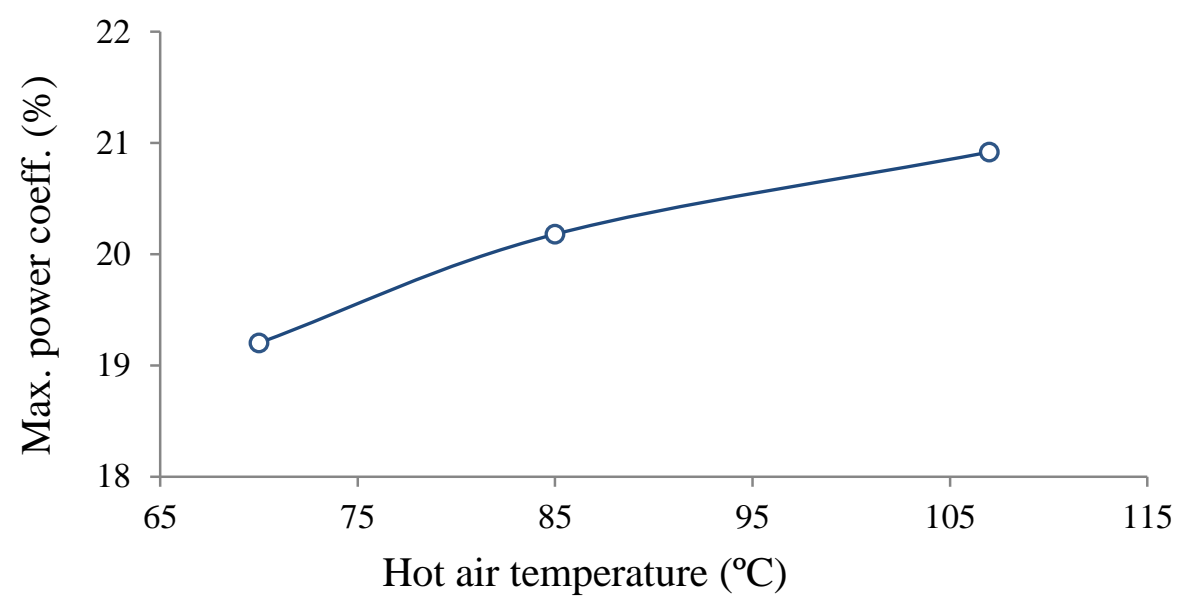

Figure 6. Effect of hot air temperature on maximum power coefficient of swirling Savonius rotor.

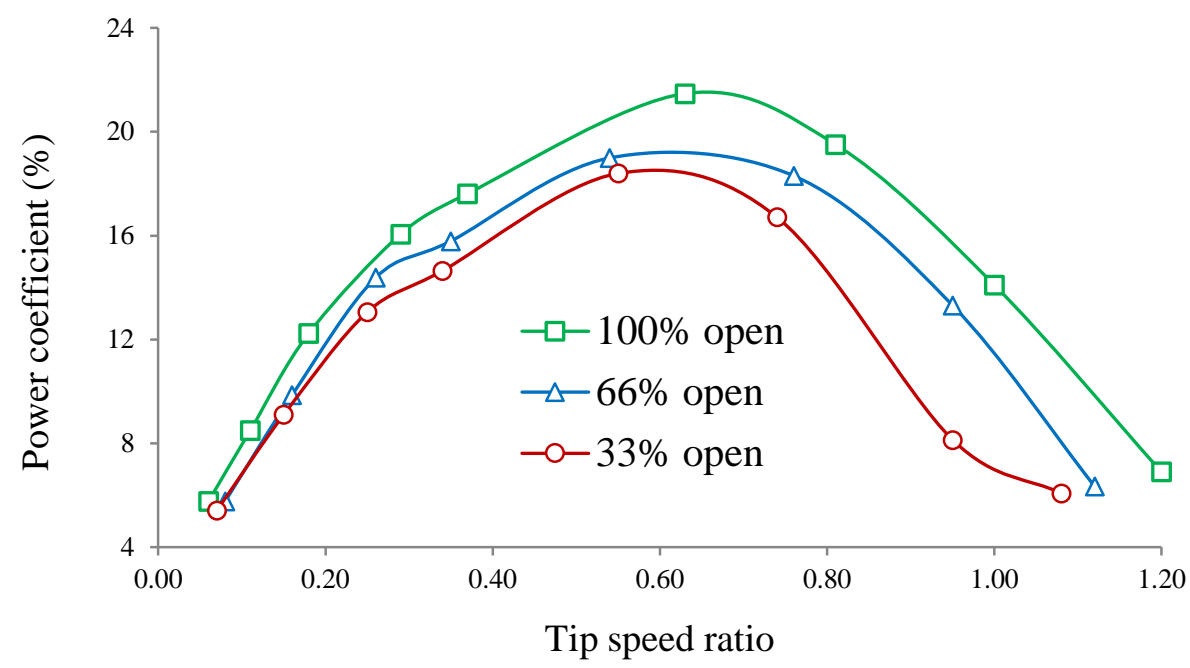

Figure 7. Power coefficient versus blade tip speed ratio curves for different hot air enclosure opening condition at $4.9 \mathrm{~m} / \mathrm{s}$ wind velocity, and $16 \mathrm{~mm}$ hot air inlet diameter.

\section{Effect of Hot Air Mass Flow Rate}

To determine the influence of hot air mass flow rate on the power coefficient, outside air enters the heat generation enclosure; consequently, the hot air flow rate was controlled by changing the enclosure inlet opening with the aid of sliding door. Rotor with 20 blade overlap ratio, $195^{\circ}$ blade arc angle, and $16 \mathrm{~mm}$ hot air inlet diameter was tested at $33 \%$, $66 \%$, and $100 \%$ opening to full capacity of enclosure inlet. The temperatures of hot air were maintained at $100^{\circ} \mathrm{C}$ for all the opening positions by controlling the heat settings of the hot plates. The power coefficients were plotted against the tip speed ratios for the three opening positions in Figure 7. It is observed from the power coefficient curves that the $C_{p}$ values at $100 \%$ open position are higher than the corresponding values of the other two positions. The maximum tip speed ratio at no load condition for $100 \%$ open position is higher (1.20) than the $66 \%$ and $33 \%$ positions, suggesting higher hot air mass flow contributing to high angular velocity of the rotor. With the increase in hot air inlet mass flow rate, the tangential 
and longitudinal velocity components of the swirling flow increase in the chamber [28]. The strong velocity profile at higher mass flow rate generates stronger torque to the rotor; subsequently, the turbine runs at faster angular speed. The maximum power coefficients versus the percentage of enclosure opening positions are presented in Figure 8. The figure clearly demonstrates an increasing trend of maximum power coefficient with the increasing mass flow rate. The reason for the enhanced performance might be the generation of stronger swirling flows at higher mass flow rate of hot air as explained above.

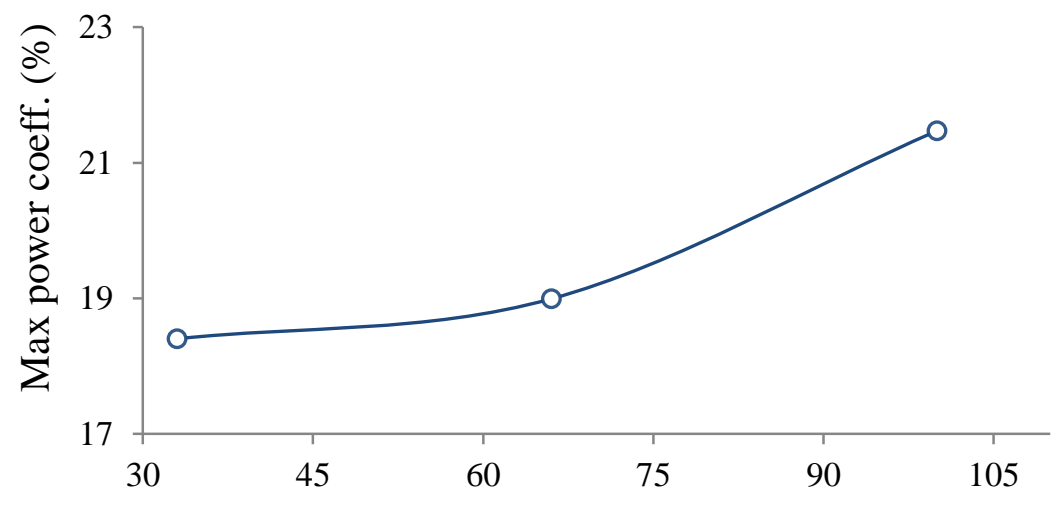

Percentage of full opening of hot air enclosure

Figure 8. Effect of hot air mass flow rate on maximum power coefficient of swirling Savonius rotor.

\section{Effect of Hot Air Inlet Diameter}

After analysing the effects of air temperature and the mass flow rate of the hot air on the performance, the optimum diameter of the inlet was determined using the full heater power of 2400 Watts and 100\% opening of enclosure inlet. This allowed obtaining the possible maximum temperature and maximum mass flow rate of hot air entering into the swirl chamber. The hot air inlet diameters of the swirling chamber in these experiments were 36 , 2516,9 , and $0 \mathrm{~mm}$. The variation of power coefficients with the blade tip speed ratio for the different hot air inlet diameter rotors at $195^{\circ}$ blade arc angle with the optimum blade overlap ratio of 0.20 (36 mm blade overlap distance) have been plotted in Figure 9. The plots indicate that power coefficient and no load tip speed ratio are higher at $16 \mathrm{~mm}$ inlet diameter than the other diameters. The strength of swirling flow depends on an optimum ratio of channel inlet to channel diameter [29]. Like the split channel geometries for fire whirl or swirling flow, where this ratio is usually around half [30], SST rotor also shows the same behaviour giving higher power coefficient at $16 \mathrm{~mm}$ inlet diameter due to higher strength of flow. Power coefficients reached maximum at around 0.60 blade tip speed ratio for $16 \mathrm{~mm}$ and $9 \mathrm{~mm}$ diameter whereas that of around 0.55 for $36 \mathrm{~mm}$ and $25 \mathrm{~mm}$.

Figure 10 presents the maximum power against the ratio of hot air inlet diameter to blade overlap distance ranging from 0 to 1 , which demonstrates that the maximum power coefficient is increasing with the increase of hot air inlet to blade overlap distance ratio and reached maximum at around 0.45 . The reason might be that when the hot air inlet diameter is reduced for the same approximate amount of mass flow rate, the inlet velocity increases, which in turn generates strong swirling flow in the chamber [28], and subsequently increases the power coefficient. The change of maximum power coefficients between the 0.45 and 0 ratio is about 33\%, which is higher than the uncertainty of power coefficient (21.7\%), so the results can be considered as conclusive. 


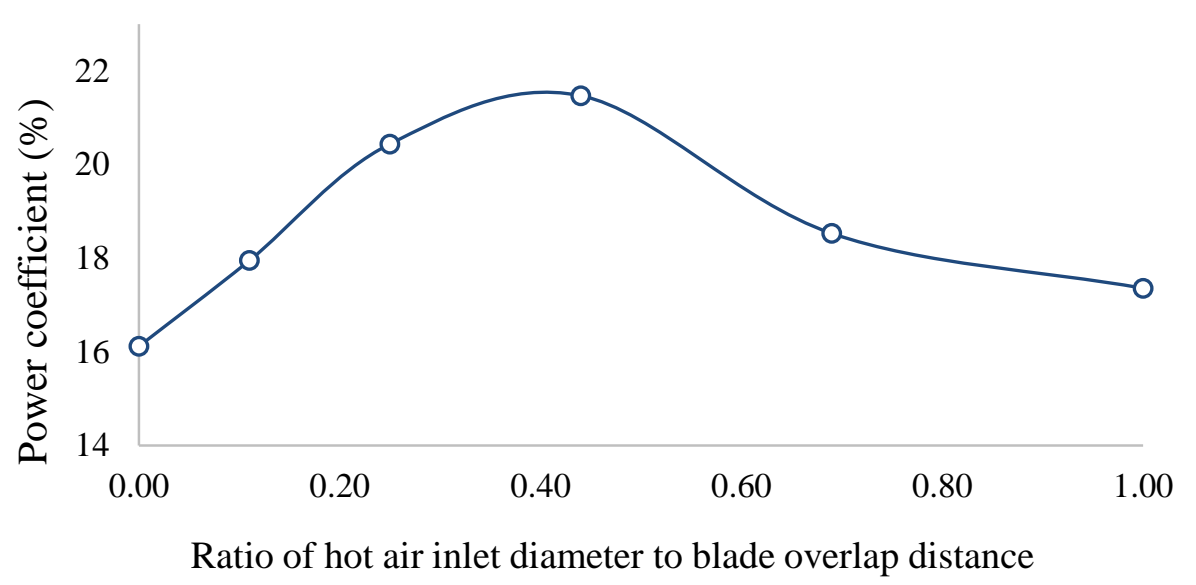

Figure 10. Maximum power coefficient with hot air inlet diameter to blade overlap distance ratio at $195^{\circ}$ blade arc angle, 0.20 blade overlap ratio, 2400 Watt heater power, and $100 \%$ enclosure inlet opening.

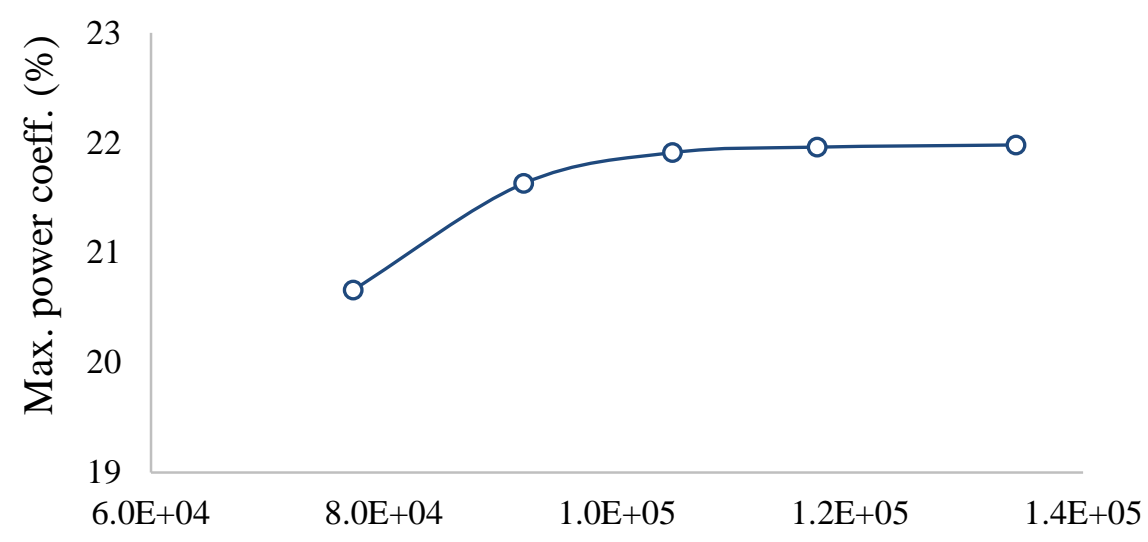

Reynold's Number

Figure 11. Variation of maximum power coefficient of optimum swirling Savonius rotor with Reynolds Number.

\section{Effect of Wind Velocity}

The optimum swirling Savonius rotor featuring 0.20 blade overlap ratio, $195^{\circ}$ blade arc angle, $16 \mathrm{~mm}$ diameter of hot air inlet and 1.06 aspect ratio rotor were tested in five different free-stream wind velocities to determine the influence of the Reynolds number on power coefficient. The wind velocities used in this experiment were 4.12, 4.90, 5.58, 6.24, and $7.15 \mathrm{~m} / \mathrm{s}$, which correspond to the Reynolds Number 77359, 92005, 104773, 117165, and 134252, respectively, based on the rotor diameter obtained by changing the damper position of the wind tunnel. Figure 11 of maximum power coefficient versus Reynolds number demonstrates that higher wind velocities yield larger power coefficients and reached almost constant value after $5.58 \mathrm{~m} / \mathrm{s}$ wind velocity (104773 Reynolds number). The maximum power coefficient at $7.15 \mathrm{~m} / \mathrm{s}$ wind velocity is $21.98 \%$, which is more than $6 \%$ higher than that of $20.66 \%$ at minimum wind velocity of $4.12 \mathrm{~m} / \mathrm{s}$ tested in this experiment. This suggests that higher angular velocity of rotor or wind velocity is favourable for the generation of strong swirling flow. Swirling strength depends on small slit width in stationary channels [30], and on larger angular speed in rotating fire whirl generator [31]. 
As the slit width of the SST rotor is relatively wider compared to that of stationary channel, the vortex strength of SST rotor mainly depends on the angular velocity of rotor. Thus, at lower wind velocity, power coefficient is lower compared to that of higher velocity.

\section{Comparison of Swirling and Conventional Savonius Turbine}

The best features of conventional and swirling Savonius turbines are compared in terms of steady state angular velocities as well as power and torque coefficients. The maximum steady state angular velocities versus applied brake torque of rotor are plotted in Figure 12. The figure demonstrates that no load maximum speed of swirling rotor is higher (357 rpm) than the conventional rotor (304 rpm). With the increase of brake torque, the differences in speed values drop and the values are almost the same before stopping. This behaviour suggests that swirling flow may not be generated at lower angular velocities but that it grows stronger with the increase of angular velocity. At lower angular velocities, only the wind turbine mechanism contributes to mechanical power output of the rotor as swirling flow may not induced in the spit chamber of the rotor. The coefficient of torque against the tip speed ratio plot is presented in Figure 13 for the optimum swirling and conventional Savonius configurations. The maximum torque coefficients for both turbines are nearly the same value of over $50 \%$, but the difference extends with higher tip speed ratio. The tip speed ratio at no load condition of swirling Savonius turbine is 1.22 , which is over $14 \%$ higher than the conventional Savonius turbine.

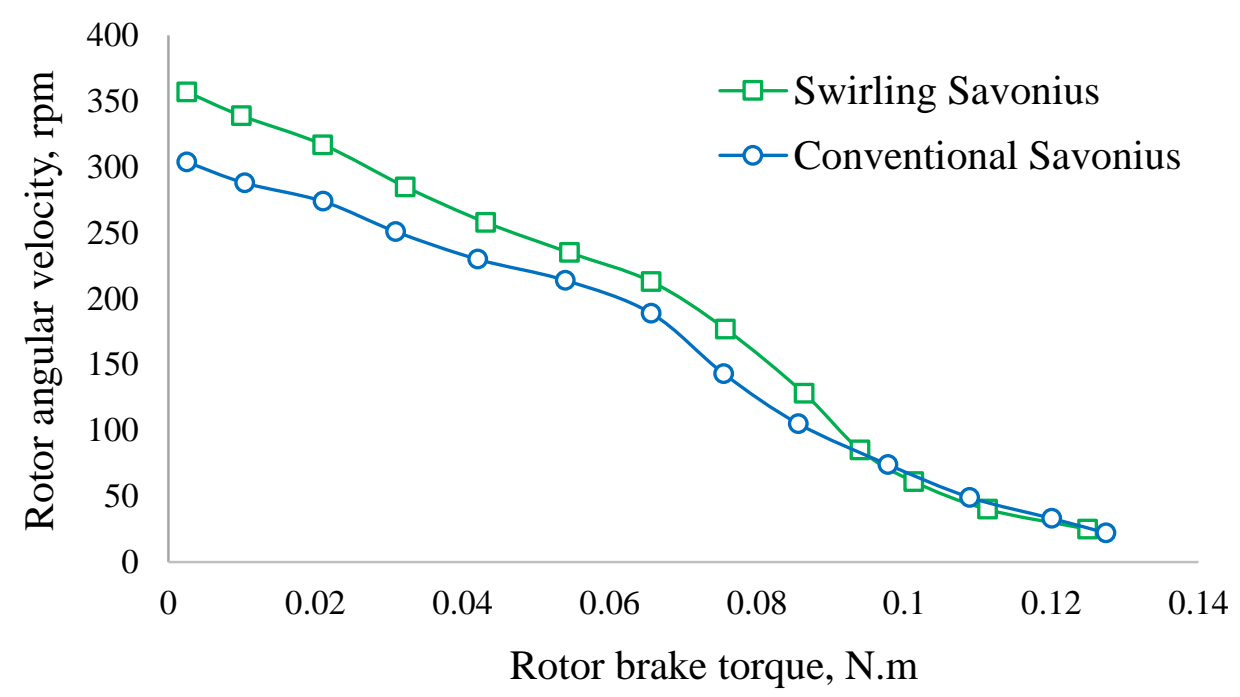

Figure 12. Steady state angular velocity of rotors at different rotor brake torques.

The plot of power coefficient versus rotor tip speed ratio for optimum swirling and conventional Savonius rotors demonstrates that for lower tip speed ratios, the values are nearly the same, but difference widens with the increase of steady state speeds of the rotor (Figure 14). The maximum power coefficient (21.66\%) was found at around 0.70 tip speed ratio and $29.83 \%$ torque coefficients for swirling turbine whereas for conventional Savonius turbine, the maximum power coefficient (17.08\%) occurred at 0.66 tip speed ratio and $25.81 \%$ torque coefficient. The increase of maximum power coefficient by $26.81 \%$ is a conclusive performance improvement of SST compared to the conventional turbine as the calculated relative uncertainty of $21.65 \%$ for power coefficient is lower than the improvement. Hybrid swirling Savonius turbine comprised of wind turbine mechanism and split channel mechanism, and both mechanisms contribute to mechanical power production. 
The superiority of SST rotor over conventional Savonius turbine in terms rotor angular speed, torque and power coefficient proved the above fact.

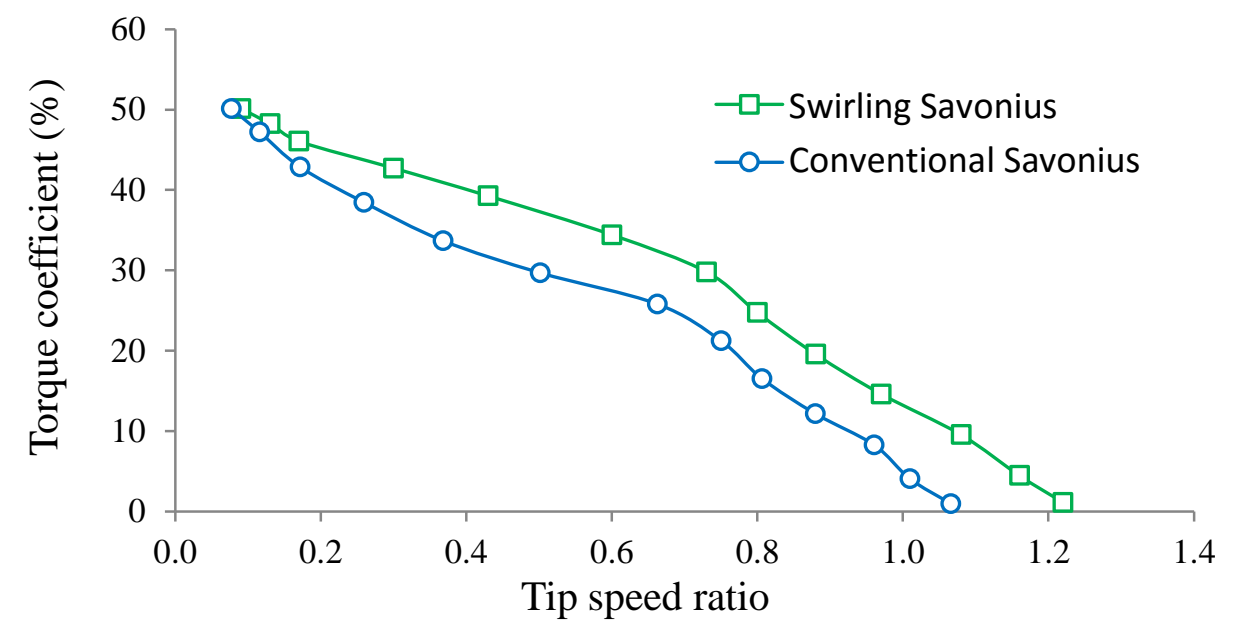

Figure 13. Variation of torque coefficients with tip speed ratio of optimum swirling and conventional Savonius rotor at $4.9 \mathrm{~m} / \mathrm{s}$ wind velocity.

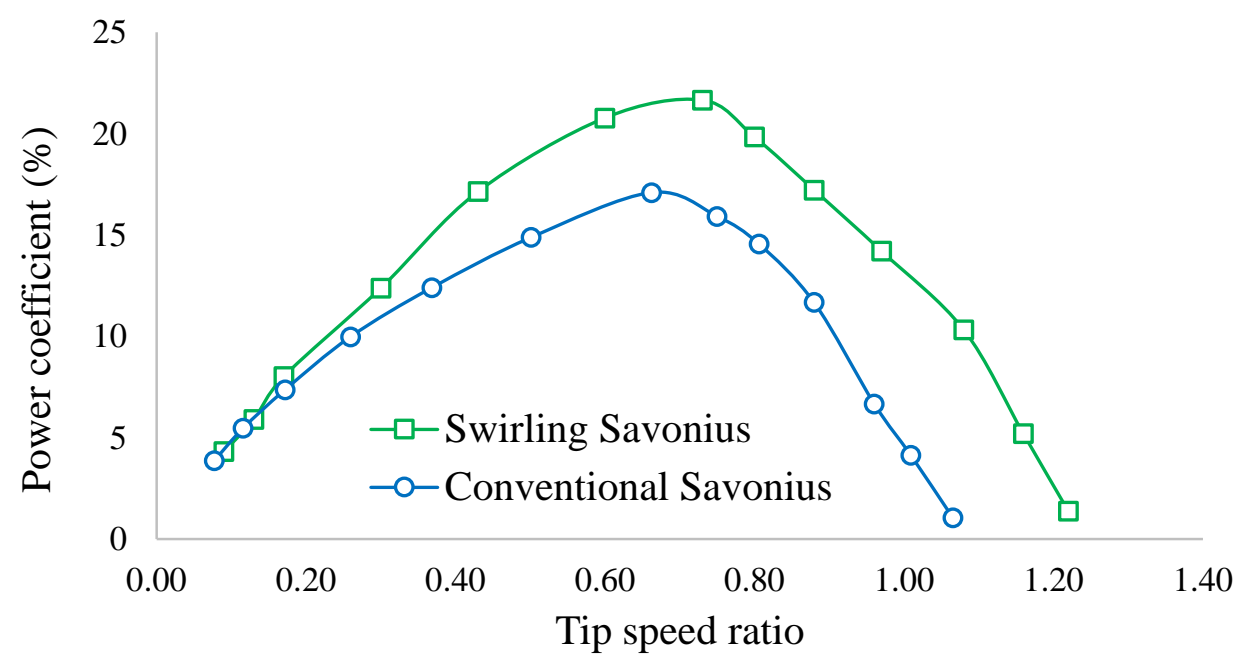

Figure 14. Variation power coefficients with tip speed ratio of optimum swirling and conventional Savonius rotor at $4.9 \mathrm{~m} / \mathrm{s}$ wind velocity.

\section{CONCLUSIONS}

The influences of flow parameters and Reynolds number on the aerodynamic coefficients have been investigated in open jet wind tunnel experiments on the optimum swirling Savonius wind turbine geometry, which is 0.20 blade overlap ratio, $195^{\circ}$ arc angle of the blades, 1.06 rotor aspect ratio with closed top end plate. The optimum geometry of the swirling turbine was also tested in different wind velocities to figure out effects of Reynolds number on performance and it was found that higher Reynolds number increases power coefficient. The results showed that higher hot air temperature and mass flow rate enhance the performance of the swirling Savonius turbine whereas power coefficient is the maximum at around 0.45 of hot air inlet diameter to blade overlap ratio, which is $16 \mathrm{~mm}$ diameter of hot air inlet in these experiments. Performance investigation of conventional Savonius turbine has also been conducted with the same experimental conditions to 
compare the performance of the two turbines. The comparison of performance between the optimum swirling and conventional Savonius turbines suggests that power coefficient of swirling turbine is improved because of the generation of swirling flow generated inside the turbine chamber and is about $27 \%$ higher than the conventional turbine. However, at lower angular velocities, the performances of swirling and Savonius turbines are almost identical.

\section{ACKNOWLEDGEMENTS}

The first author would like to express his appreciation to the Australian government for the financial support to pursue his Doctoral study through the International Postgraduate Research Scholarship (IPRS) and Australian Postgraduate Award (APA).

\section{REFERENCES}

[1] Omer AM. Built environment: relating the benefits of renewable energy technologies. International Journal of Automotive and Mechanical Engineering. 2012;5:561-75.

[2] Haruni AMO. A stand-alone hybrid power system with energy storage. Tasmania: University of Tasmania; 2013.

[3] Damak A, Driss Z, Abid MS. Experimental investigation of helical Savonius rotor with a twist of $180^{\circ}$. Renewable Energy. 2013;52:136-42.

[4] Supeni EE, Epaarachchi JA, Islam MM, Lau KT. Development of Artificial Neural Network Model in Predicting Performance of the Smart Wind Turbine Blade. Journal of Mechanical Engineering and Sciences. 2014;6:734-45.

[5] Torresi M, Benedittisa FAD, Fortunato B, Camporeale SM. Performance and flow field evaluation of a Savonius rotor tested in a wind tunnel. Energy Procedia. 2014;45:207-16.

[6] Menet JL. Aerodynamic behaviour of a new type of slow-running VAWT. In: Peinke J, Schaumann P, Barth S, editors. Wind energy proceedings of the euromech colloquium. Berlin Heidelberg: Springer; 2007. p. 235-9.

[7] Sheldahl RE, Blackwell BF, Feltz LV. Wind tunnel performance data for two- and three-bucket Savonius rotors. Journal of Energy. 1978;2:160-4.

[8] Valdès LC, Darque J. Design of wind-driven generator made up of dynamos assembling. Renewable Energy. 2003;28:345-62.

[9] Savonius SJ. The S-rotor and its applications. Mechanical Engineering. 1931;53:333-8.

[10] Fujisawa N, Gotoh F. Experimental study on the aerodynamic performance of a Savonius rotor. Journal of Solar Energy Engineering. 1994;116:148-52

[11] Kamoji MA, Prabhu SV, Kedare SB. Experimental investigations on the performance of conventional Savonius rotor under static and dynamic conditions. 3rd International Conference on Fluid Mechanics and Fluid Power. 2006.

[12] Akwa JV, Júnior GAdS, Petry AP. Discussion on the verification of the overlap ratio influence on performance coefficients of a Savonius wind rotor using computational fluid dynamics. Renewable Energy. 2012;38:141-9.

[13] Modi VJ, Fernando MSUK. On the Performance of the Savonius Wind Turbine. Journal of Solar Energy Engineering. 1989;111:71-81.

[14] Manwell JF, McGowan JG, Rogers AL. Wind energy explained: theory, design and application. Second ed. Great Britain: John Wiley \& Sons Ltd.; 2009. 
[15] Hayashi T, Li Y, Hara Y. Wind tunnel tests on a different phase three-stage Savonius rotor. JSME International Journal Series B. 2005;48:9-16.

[16] Kamoji MA, Kedare SB, Prabhu SV. Performance tests on helical Savonius rotors. Renewable Energy. 2009;34:521-9.

[17] Saha UK, Rajkumar MJ. On the performance analysis of Savonius rotor with twisted blades. Renewable Energy. 2006;31:1776-88.

[18] Ushiyama I, Nagai H, Shionoda J. Experimentally determining the optimum design configuration for Savonius rotors. Transaction of the Japan Society of Mechanical Engineers Series B. 1986;52:2973-81

[19] Irabu K, Roy JN. Characteristics of wind power on Savonius rotor using a guidebox tunnel. Experimental Thermal and Fluid Science. 2007;32:580-6.

[20] Ogawa T, Yoshida H, Yokota Y. Development of rotational speed control systems for a Savonius-type wind turbine. Journal of Fluids Engineering. 1989;111:53-8.

[21] Altan BD, Atilgan M, Özdamar A. An experimental study on improvement of a Savonius rotor performance with curtaining. Experimental Thermal and Fluid Science. 2008;32:1673-8.

[22] Al-Faruk A, Sharifian AS, Al-Atresh SR. Numerical investigation of performance of a new type of Savonius turbine.18th Australasian Fluid Mechanics Conference. Launceston, Australia. 2012.

[23] Al-Faruk A, Sharifian A. Influence of blade overlap and blade angle on the aerodynamic coefficients in vertical axis swirling type Savonius wind turbine. 19th Australasian Fluid Mechanics Conference. Melbourne, Australia. 2014.

[24] Menet JL. A double-step Savonius rotor for local production of electricity: a design study. Renewable Energy. 2004;29:1843-62.

[25] Moutsoglou A, Weng Y. Performance tests of a Benesh wind turbine rotor and a Savonius rotor. Wind Engineering. 1995;19:349-62.

[26] Bevington PR, Robinson DK. Data reduction and error analysis for the physical sciences. 3rd ed. Crawfordsville, IN: McGraw-Hill; 2003.

[27] Hau E. Wind turbines fundamental technologies, application and economics. New Delhi, India: Springer-Verlag Berlin Heidelberg; 2006.

[28] Al-Atresh S, Sharifian AS, Al-Faruk A. The effect of inlet velocity and temperature on the strength of swirling induced by a split channel: A CFD approach. 18th Australasian Fluid Mechanics Conference. Launceston, Australia. 2012.

[29] Kuwana K, Morishita S, Dobashi R, Chuah KH, Saito K. The burning rate's effect on the flame length of weak fire whirls. Combustion Institute. 2011.

[30] Al-Atresh SR, Sharifian AS, Wandel AP. The effect of the width and number of gaps on the characteristics of swirl flow induced natually inside split channel using hot air inlet. International Journal of Materials, Mechanics and Manufacturing. 2014;2:339-44.

[31] Emmons HW, Ying S-J. The fire whirl. Symposium (International) on Combustion. 1967;11:475-88. 